BMJ Open

Diabetes

Research

\& Care

\section{Impact of sex and age on the performance of FINDRISC: the HUNT Study in Norway}

To cite: Jølle A, Midthjell K, Holmen J, et al. Impact of sex and age on the performance of FINDRISC: the HUNT Study in Norway. BMJ Open Diabetes Research and Care 2016;4:e00217. doi:10.1136/bmjdrc-2016000217

- Additional material is available. To view please visit the journal online (http://dx.doi.org/10.1136/ bmjdrc-2016-000217).

Received 24 February 2016 Accepted 14 April 2016

\section{CrossMark}

For numbered affiliations see end of article.

Correspondence to

Dr Anne Jølle;

Anne.jolle@ntnu.no

\section{ABSTRACT}

Objective: The Finnish Diabetes Risk Score

(FINDRISC) is recommended as a screening tool for diabetes risk. However, there is a lack of well-powered studies examining the performance of FINDRISC by sex and age. We aim to estimate, by sex and age, the prevalence of elevated FINDRISC and positive predictive value (PPV) of FINDRISC for identifying impaired glucose metabolism (IGM) in a general Norwegian population.

Research design and methods: We estimated the prevalence of elevated FINDRISC ( $\geq 15)$ among 47694 adults in the third survey of the Nord-Trøndelag Health Study (HUNT3, 2006-08). Among 2559 participants who participated in oral glucose tolerance testing, we estimated the PPV of elevated FINDRISC for identifying unknown prevalent diabetes and other forms of IGM.

Results: The prevalence of elevated FINDRISC was $12.1 \%$ in women, $9.6 \%$ in men, and increased from $1.5 \%$ at age $20-39$ to $25.1 \%$ at age $70-79$ years. The PPVs of elevated FINDRISC were $9.8 \%$ for diabetes, $16.9 \%$ for impaired glucose tolerance, $8.2 \%$ for impaired fasting glucose, and $34.9 \%$ for any form of IGM. The PPV for IGM was lower in women $(31.2 \%)$ than in men $(40.4 \%)$, and increased from $19.1 \%$ at age $20-39$ to $55.5 \%$ at age $\geq 80$ years.

Conclusions: FINDRISC identified more women than men as high-risk individuals for diabetes. FINDRISC had a high PPV for detecting prevalent IGM, and the PPV was higher in men than in women and in the older individuals. Our data indicate that the impact of sex and age on diabetes risk is not fully captured by FINDRISC, and that refinements to it might improve diabetes prediction.

The worldwide number of people with type 2 diabetes is estimated to increase from 382 million in 2013 to 592 million in $2035,{ }^{1}$ and more than $30 \%^{2}$ of cases are undiagnosed since mild hyperglycemia carries no specific symptoms. Type 2 diabetes is partly preventable by lifestyle intervention, ${ }^{3-5}$ but effective intervention is not easy to implement on a population level. Cost-effective, non-invasive and reliable risk screening tools for diabetes are important for early diagnosis, and

\section{Key messages}

- In a general adult population in Norway, FINDRISC $\geq 15$ classified $11 \%$ as being at high risk for diabetes, and this proportion was higher among women than men.

- An elevated FINDRISC had a positive predictive value of $10 \%$ for prevalent diabetes and $35 \%$ for diabetes and other forms of impaired glucose metabolism combined. The positive predictive value was higher in men than women and in older than younger individuals.

- Our results indicate that the impact of sex and age on diabetes risk is not fully captured by FINDRISC, and that refinements to it might improve diabetes prediction.

likewise to recognize people at high risk of developing type 2 diabetes who can be targeted in sustainable prevention strategies.

One recommended and widely used risk screening tool is the Finnish Diabetes Risk Score (FINDRISC), an eight-item questionnaire including age; body mass index (BMI); waist circumference; physical activity; daily consumption of fruits, berries, or vegetables; history of antihypertensive drug treatment; history of high blood glucose; and family history of diabetes. ${ }^{67}$ The different items are weighted into a total score ranging from 0 to 26 points. ${ }^{6}$ In the original Finnish study population, a score of $\geq 15$ was associated with a high risk of developing type 2 diabetes within the subsequent 10 years. ${ }^{6} 8$

FINDRISC has been evaluated in several studies in Europe and other regions and is shown to be a reliable predictor for future diabetes and prevalent undiagnosed diabetes in most of these studies. ${ }^{7} 9-18$ Few of these studies have been population-based. Consequently, little is known about the prevalence of elevated FINDRISC in the general population and by age or sex, although some studies have reported a higher prevalence of elevated FINDRISC in women than in 
men. ${ }^{8} 1719$ Such information is essential in order to evaluate the extent of healthcare needed for follow-up of high-risk individuals if FINDRISC is incorporated as a screening tool at the population level. Furthermore, it is not clear whether an elevated FINDRISC score carries a similar risk of diabetes in younger compared with older adults, and in men compared with women. However, two studies reported that among people with elevated FINDRISC, undiagnosed diabetes and high-risk conditions of diabetes such as metabolic syndrome, fasting plasma glucose, 2 hours plasma glucose, lipids, and blood pressure were more common in men than in women. ${ }^{817}$ We aimed to estimate the prevalence of elevated FINDRISC by sex and age groups, and to determine how well elevated FINDRISC identifies prevalent impaired glucose tolerance (IGT), impaired fasting glucose (IFG), and diabetes, by sex and age groups, in a general population of adult Norwegians.

\section{RESEARCH DESIGN AND METHODS}

\section{Study population}

The Nord-Trøndelag Health Study (HUNT) is a population-based study in Nord-Trøndelag County, Norway. All inhabitants aged $\geq 20$ years were invited to participate in three cross-sectional surveys during a 22-year period: HUNT1 (1984-1986), HUNT2 (19951997), and HUNT3 (2006-2008). The HUNT includes comprehensive questionnaires, interviews, clinical measurements, and a collection of biological samples. Details about the study are described elsewhere. ${ }^{20}$ The population of Nord-Trøndelag is considered representative of Norway, except that the county lacks big cities and that the mean income and education level and proportion of immigrants are somewhat lower than the Norwegian average ${ }^{20}$ In this study, we used data from the HUNT3 survey, in which 50806 individuals $(54.1 \%$ of those invited) participated between October 2006 and June 2008.

\section{Diabetes and FINDRISC in the HUNT3 survey}

All participants completed a self-administered questionnaire which included information on previously known diabetes and the FINDRISC items. Weight and height were measured with the participants wearing light clothes without shoes, and BMI was calculated as weight (in $\mathrm{kg}$ ) divided by the squared value of height (in meters). Waist circumference was measured horizontally at the level of the umbilicus with a non-stretchable band, the participant standing, and the arms hanging relaxed. FINDRISC was calculated at the screening site using the following variables and scoring system: age in years ( $<45,0 ; 45$ to 54,$2 ; 55$ to 64,3 ; and $\geq 65,4$ points); BMI in $\mathrm{kg} / \mathrm{m}^{2}(<25,0$; 25 to 30,$1 ;>30,3$ points); waist circumference in $\mathrm{cm}$ (men: $<94,0 ; 94$ to 102,3 ; $>102,4$ points, and women: $<80,0 ; 80$ to 88,$3 ;>88,4$ points); physical activity $(\geq 30 \mathrm{~min} /$ day, $0 ;<30 \mathrm{~min} /$ day, $\quad 2$ points); daily consumption of fruits, berries, or vegetables (yes, 0 ; no, 1 point); ever regular use of antihypertensive medication (no, 0 ; yes, 2 points); history of high blood glucose measurement (no, 0 ; yes, 5 points); and family history of diabetes (no, 0 ; second but no first degree relative, 3; first degree relative, 5 points). Participants without previously known diabetes with FINDRISC $\geq 15$ received information about their 10 -year risk of developing type 2 diabetes and were invited to join the HUNT Diabetes in Europe-Prevention using Lifestyle, physical Activity and Nutritional intervention (DE-PLAN) Study. Glucose level was measured in nonfasting serum samples in all participants, and participants without known diabetes who had a non-fasting serum glucose $\geq 9.0 \mathrm{mmol} / \mathrm{L}$ were recommended to consult their general practitioner.

The HUNT3 survey also included information on other clinical characteristics associated with diabetes. The self-administered questionnaire included information on smoking habits and history of cardiovascular diseases. Blood pressure and resting heart rate were measured three times while the participants were sitting, at $1 \mathrm{~min}$ intervals using an automated blood pressure monitor based on oscillometry (Dinamap 845XT; Critikon, Tampa, Florida, USA). The mean values of the second and third measurements were used in the analyses. Concentrations of total cholesterol, high-density lipoprotein (HDL) cholesterol, triglycerides, and creatinine were measured in non-fasting serum samples.

\section{The HUNT DE-PLAN Study}

The HUNT3 study cohort was 1 of 25 European cohorts included in the DE-PLAN Study where the main goal was to improve the capability to prevent diabetes in Europe. $^{21}$ All HUNT3 participants with FINDRISC $\geq 15$ and no previously known diabetes were invited to the HUNT DE-PLAN Study, which included a baseline examination followed by a diabetes prevention programme. In the present study, we used information from the baseline examination, which included a questionnaire and clinical and laboratory measurements. After overnight fasting, participants underwent an oral glucose tolerance test (OGTT) if they had a fasting plasma-referenced capillary glucose level $<8.5 \mathrm{mmol} / \mathrm{L}$. Participants with a fasting capillary glucose level $\geq 8.5 \mathrm{mmol} / \mathrm{L}$ had a fasting blood sample drawn for serum glucose measurement, but were not offered an OGTT since they were highly likely to have diabetes that could be classified on the basis of the fasting serum glucose level alone. The OGTT was performed by giving the participants $75 \mathrm{~g}$ of glucose dissolved in $300 \mathrm{~mL}$ of water, to be consumed within $5 \mathrm{~min}$. Glucose levels were measured in serum samples drawn before (fasting) and $120 \mathrm{~min}$ after the glucose ingestion. Whole blood glycated hemoglobin (HbAlc) was measured in all the participants.

According to the 1999 WHO criteria, ${ }^{22}$ diabetes was defined as fasting serum glucose $\geq 7.0 \mathrm{mmol} / \mathrm{L}$ or $120 \mathrm{~min}$ serum glucose $\geq 11.1 \mathrm{mmol} / \mathrm{L}$. IGT was defined 
as $120 \mathrm{~min}$ serum glucose of $7.8-11.0 \mathrm{mmol} / \mathrm{L}$, but no diabetes. IFG was defined as fasting serum glucose of 6.1-6.9 mmol/L in people who did not have diabetes or IGT. Impaired glucose metabolism (IGM) was defined as diabetes, IGT, or IFG. In an additional analysis, we included HbAlc of $\geq 6.5 \%(\geq 48 \mathrm{mmol} / \mathrm{mol})$ as an alternative and sufficient criterion for diabetes, based on diagnostic criteria endorsed by the WHO in $2011 .^{23}$

\section{Laboratory measurements}

Serum glucose was analyzed by hexokinase/G-6-PDH methodology, total cholesterol by an enzymatic cholesterol esterase method, HDL cholesterol by an accelerator selective detergent method, triglycerides by a glycerol phosphate oxidase method, and creatinine by an alkaline picrate method (all Abbott, Clinical Chemistry, Illinois, USA). Plasma-referenced capillary glucose was measured at the screening site by glucose dehydrogenase methodology using a HemoCue photometer (HemoCue AB, Ängelholm, Sweden). The other blood samples were analyzed on an Architect ci8200 (Abbott Diagnostics, Longford, Ireland) at Levanger Hospital, Nord-Trøndelag Hospital Trust. HbAlc was measured immunoturbidimetrically by using a microparticle agglutination inhibition method (Multigent, Abbott Laboratories, Illinois, USA).

\section{Statistical analysis}

Among 50806 participants in the HUNT3 survey, we excluded 2290 persons with previously known diabetes and 124 with missing information on self-reported diabetes, leaving 48392 participants eligible for this study. A total of $47694(98.3 \%)$ had information on all FINDRISC items, and for these we calculated the prevalence (with a 95\% CI) of elevated FINDRISC $(\geq 15)$ overall and by categories of sex and age. As a measure of the associations of sex and age with elevated FINDRISC, we estimated prevalence ratios (with 95\% CIs) of elevated FINDRISC according to sex and age groups by using log-binomial regression analysis. To assess which FINDRISC components explained the sex and age differences in prevalence, we examined the distribution of each FINDRISC component by sex and age groups.

Among 5297 participants with elevated FINDRISC and no previously known diabetes, 2633 (49.7\%) participated in the baseline examination of the HUNT DE-PLAN Study, and fasting serum glucose was measured in 2580 of them. Among 2433 HUNT DE-PLAN participants with fasting serum glucose $<7.0 \mathrm{mmol} / \mathrm{L}, 2412(99.1 \%)$ also had $120 \mathrm{~min}$ glucose measured; they were included and weighted in the statistical analyses to represent all 2433 participants with fasting serum glucose $<7.0 \mathrm{mmol} / \mathrm{L}$. All 147 participants with fasting serum glucose $\geq 7.0 \mathrm{mmol} / \mathrm{L}$ were included in the statistical analyses, as they could be classified with diabetes on the basis of the fasting serum sample only. Among these 2559 HUNT DE-PLAN participants, we estimated the positive predictive value (PPV, with 95\% CI) of elevated FINDRISC for identifying diabetes, IGT, IFG, and any IGM overall and by categories of sex and age.

In additional analyses, we similarly estimated the prevalence of mildly (score 15-19) and markedly (score $\geq 20$ ) elevated FINDRISC, and the PPV for different forms of IGM within each of these groups.

Since differences in the participation rate between sex and age groups in HUNT3 could influence the overall prevalence estimate of elevated FINDRISC, we also estimated this prevalence using probability weights to account for differences in sex and age distribution between HUNT3 participants and the total adult ( $\geq 20$ years of age) population of Nord-Trøndelag. For this purpose, we used information on the population in Nord-Trøndelag by sex and 10-year age categories on 1 January 2007, as provided by Statistics Norway. ${ }^{24}$

To evaluate the possibility of selection bias due to nonparticipation in the HUNT DE-PLAN Study, we calculated the participation rate in the HUNT DE-PLAN Study by sex and age groups and examined whether FINDRISC differed between participants and nonparticipants of the HUNT DE-PLAN Study.

The statistical analyses were performed using IBM SPSS Statistics V.22.0 for Windows (Armonk, New York, USA), and Stata V.13.1 for Windows (StataCorp, College Station, Texas, USA).

\section{Ethics}

All participants signed an informed consent. The study was approved by the Regional Committee for Medical and Health Research Ethics of Central Norway.

\section{RESULTS}

\section{Prevalence of elevated FINDRISC}

Among 47694 individuals with information available on all FINDRISC items, the mean (SD) FINDRISC was 8.8 (4.5) overall, 9.1 (4.6) in women, and 8.4 (4.5) in men. Table 1 shows participant characteristics overall, by sex and by FINDRISC (0-14 and 15-26). As expected, participants with FINDRISC $\geq 15$ had higher levels of most cardiovascular risk factors including lipids, blood pressure, and non-fasting serum glucose.

The prevalence of elevated FINDRISC was $11.0 \%$, and it was higher in women $(12.1 \%)$ than in men $(9.6 \%$; table 2). The score components that led to the higher prevalence among women were waist circumference, family history of diabetes, and previously measured high blood glucose (see online supplementary table S1). The prevalence of elevated FINDRISC increased with age and was 17 times higher at $70-79$ years $(24.9 \%)$ compared with 20-39 years of age (1.5\%; table 2). All score components contributed to the higher prevalence at older ages, except intake of fruit, berries, and vegetables (see online supplementary table $\mathrm{S} 1$ ). The prevalence of elevated FINDRISC by combinations of sex and age group is shown in online supplementary figure $\mathrm{S} 1$. We separately examined the prevalence of mildly ${ }^{15-19}$ and 
Table 1 Characteristics of the 47694 participants who had information on all FINDRISC items, by FINDRISC and sex, given as mean (SD) unless otherwise noted

\begin{tabular}{|c|c|c|c|c|c|c|}
\hline \multirow[b]{2}{*}{ FINDRISC variables } & \multicolumn{3}{|l|}{ Women } & \multicolumn{3}{|l|}{ Men } \\
\hline & $\begin{array}{l}\text { Total } \\
(n=26140)\end{array}$ & $\begin{array}{l}0-14 \\
(n=2969)\end{array}$ & $\begin{array}{l}15-26 \\
(n=3171)\end{array}$ & $\begin{array}{l}\text { Total } \\
(n=21554)\end{array}$ & $\begin{array}{l}0-14 \\
(n=19486)\end{array}$ & $\begin{array}{l}15-26 \\
(n=2068)\end{array}$ \\
\hline Age (years) & $52.2(16.2)$ & $50.5(15.9)$ & $64.7(12.5)$ & $53.0(15.6)$ & $51.8(15.5)$ & $64.1(10.9)$ \\
\hline Waist circumference $(\mathrm{cm})$ & $89.9(12.5)$ & $88.3(11.9)$ & $101.5(11.1)$ & $97.0(10.3)$ & $95.9(9.9)$ & $107.4(8.6)$ \\
\hline BMI $\left(\mathrm{kg} / \mathrm{m}^{2}\right)$ & $26.8(4.8)$ & $26.2(4.5)$ & $31.1(4.6)$ & $27.4(3.7)$ & $27.0(3.5)$ & $30.9(3.4)$ \\
\hline Physical activity $\geq 30 \mathrm{~min} /$ day (\%) & 78.1 & 80.9 & 57.4 & 75.1 & 77.6 & 51.7 \\
\hline Daily fruit, berries, or vegetables (\%) & 70.2 & 70.7 & 66.8 & 50.2 & 50.5 & 47.7 \\
\hline Ever treated for hypertension (\%) & 18.9 & 13.2 & 59.9 & 19.2 & 15.0 & 59.3 \\
\hline Ever measured high blood glucose (\%) & 5.3 & 2.5 & 25.4 & 3.0 & 1.1 & 21.0 \\
\hline First degree relative with diabetes (\%) & 24.2 & 17.5 & 72.2 & 20.6 & 15.4 & 69.9 \\
\hline Second degree relative with diabetes (\%) & 34.7 & 31.6 & 56.5 & 28.1 & 25.3 & 54.0 \\
\hline \multicolumn{7}{|l|}{ Other variables } \\
\hline Non-fasting serum glucose (mmol/L) & $5.3(1.1)$ & $5.3(0.9)$ & $5.8(1.5)$ & $5.6(1.3)$ & $5.5(1.2)$ & $6.1(1.7)$ \\
\hline Total serum cholesterol (mmol/L) & $5.6(1.1)$ & $5.5(1.1)$ & $5.8(1.1)$ & $5.5(1.1)$ & $5.5(1.1)$ & $5.4(1.1)$ \\
\hline Serum HDL cholesterol (mmol/L) & $1.5(0.4)$ & $1.5(0.4)$ & $1.4(0.3)$ & $1.2(0.3)$ & $1.2(0.3)$ & $1.1(0.3)$ \\
\hline Non-fasting serum triglycerides (mmol/L) & $1.4(0.8)$ & $1.4(0.8)$ & $1.8(0.9)$ & $1.8(1.1)$ & $1.8(1.1)$ & $2.1(1.1)$ \\
\hline Serum creatinine $(\mu \mathrm{mol} / \mathrm{L})$ & $75(15)$ & $74(14)$ & $80(20)$ & $90(18)$ & $89(18)$ & $95(22)$ \\
\hline Systolic blood pressure (mm Hg) & $128(19)$ & $126(19)$ & $139(21)$ & $133(17)$ & $133(16)$ & 140 (19) \\
\hline Diastolic blood pressure $(\mathrm{mm} \mathrm{Hg})$ & $71(11)$ & $71(11)$ & $74(11)$ & $76(11)$ & $76(11)$ & $80(11)$ \\
\hline Waist/hip ratio & $0.87(0.07)$ & $0.86(0.07)$ & $0.92(0.06)$ & $0.94(0.06)$ & $0.93(0.06)$ & $1.00(0.05)$ \\
\hline Daily cigarette smoking (\%) & 19.3 & 19.9 & 14.9 & 14.4 & 14.6 & 12.4 \\
\hline Cardiovascular disease (\%) & 5.3 & 4.0 & 14.7 & 9.9 & 8.4 & 24.4 \\
\hline
\end{tabular}

BMI, body mass index; FINDRISC, Finnish Diabetes Risk Score; HDL, high-density lipoprotein.

markedly $(\geq 20)$ elevated FINDRISC. For both outcomes, the prevalence was higher among women than men and increased considerably with age (table 2 ).

In an additional analysis, we weighted the study participants to account for differences in sex and age distributions between HUNT3 participants and the total adult population of Nord-Trøndelag. This analysis yielded slightly lower prevalence estimates; the prevalence of elevated FINDRISC $(\geq 15)$ was $9.6 \%$ (95\% CI $9.4 \%$ to $9.9 \%$ ), and the prevalence of markedly elevated FINDRISC ( $\geq 20)$ was $1.0 \%$ (95\% CI $1.0 \%$ to $1.1 \%$ ).

\section{PPV of elevated FINDRISC}

Among 5297 people with elevated FINDRISC who were invited to the HUNT DE-PLAN Study, the participation rate in the baseline HUNT DE-PLAN examination was similar among women $(49 \%)$ and men $(51 \%)$, and broadly similar across age groups $(48-58 \%)$, except that among people $\geq 80$ years of age only $31 \%$ participated. Among invitees to the HUNT DE-PLAN Study, FINDRISC was similar in participants (median 16, IQR 15-18) and non-participants (median 16, IQR 15-18).

Among the 2559 participants without previously known diabetes who had their glucose tolerance examined, the PPV of elevated FINDRISC was $9.8 \%$ for prevalent diabetes, $16.9 \%$ for IGT, $8.2 \%$ for IFG, and $34.9 \%$ for any of these forms of IGM (table 3). The PPV for IGM was lower in women $(31.2 \%)$ than in men $(40.4 \%)$. Specifically, the PPVs for diabetes and IFG were lower in women than in men, whereas the PPVs for IGT were similar. The PPV for IGM increased strongly and continuously with age from $19.1 \%$ at age $20-39$ to $55.5 \%$ at age $\geq 80$ years. Specifically, the PPVs for diabetes and IGT increased with age, whereas the PPV for IFG was highest at 50-59 years of age. The PPVs for different forms of IGM by combinations of sex and age group are shown in figure 1.

Out of the 2559 HUNT DE-PLAN participants with elevated FINDRISC, 2545 (99.5\%) had information available on all FINDRISC items. Among these, 2233 $(87.7 \%)$ had mildly elevated ${ }^{15-19}$ and $312(12.3 \%)$ had markedly $(\geq 20)$ elevated FINDRISC. The PPVs for diabetes and other forms of IGM were higher among people with markedly elevated (see online supplementary table S2) compared with mildly (see online supplementary table S3) elevated FINDRISC. However, the increases in PPV for diabetes and IGT by age were retained for mildly and markedly elevated FINDRISC. The lower PPV among women than men was also retained among people with mildly elevated FINDRISC, whereas no convincing relationship between sex and PPV was observed for markedly elevated FINDRISC.

Using $\mathrm{HbAlc} \geq 6.5 \%(\geq 48 \mathrm{mmol} / \mathrm{mol})$ as an alternative criterion for diabetes, slightly different PPVs were observed for elevated FINDRISC: diabetes, $12.5 \%(95 \%$ CI $11.3 \%$ to $13.9 \%$ ); IGT, $15.6 \%$ (95\% CI $14.3 \%$ to $17.1 \%$ ); IFG, $7.7 \%$ (95\% CI $6.7 \%$ to $8.8 \%$ ); and IGM, $35.8 \%$ (95\% CI $34.0 \%$ to $37.7 \%$ ). Corresponding PPVs for markedly elevated FINDRISC were: diabetes, 19.8\% (95\% CI $15.7 \%$ to $24.6 \%$ ); IGT, $22.1 \%$ (95\% CI $17.8 \%$ to $27.1 \%$ ); IFG, $9.3 \%$ (95\% CI $6.5 \%$ to $13.1 \%$ ); and IGM, $51.2 \%$ (95\% CI $45.7 \%$ to $56.8 \%$ ). 
Table 2 The prevalence of Finnish Diabetes Risk Score (FINDRISC) $\geq 15$, FINDRISC 15 to 19 and FINDRISC $\geq 20$, overall and for sex and age groups

\begin{tabular}{|c|c|c|c|c|c|c|c|c|c|c|c|c|}
\hline & \multicolumn{4}{|c|}{ FINDRISC $\geq 15$} & \multicolumn{4}{|c|}{ FINDRISC 15-19 } & \multicolumn{4}{|c|}{ FINDRISC $\geq \mathbf{2 0}$} \\
\hline & \multicolumn{2}{|c|}{ Prevalence (\%) } & \multicolumn{2}{|c|}{ Prevalence ratio } & \multicolumn{2}{|c|}{ Prevalence (\%) } & \multicolumn{2}{|c|}{ Prevalence ratio } & \multicolumn{2}{|c|}{ Prevalence (\%) } & \multicolumn{2}{|c|}{ Prevalence ratio } \\
\hline & & $95 \% \mathrm{Cl}$ & & $95 \% \mathrm{Cl}$ & & $95 \% \mathrm{Cl}$ & & $95 \% \mathrm{Cl}$ & & $95 \% \mathrm{Cl}$ & & $95 \% \mathrm{Cl}$ \\
\hline $\begin{array}{l}\text { Overall } \\
\text { Sex }\end{array}$ & 11.0 & 10.7 to 11.3 & & & 9.8 & 9.5 to 10.1 & & & 1.2 & 1.1 to 1.3 & & \\
\hline Men & 9.6 & 9.2 to 10.0 & 1.00 & Reference & 8.7 & 8.3 to 9.1 & 1.00 & Reference & 0.9 & 0.8 to 1.1 & 1.00 & Reference \\
\hline Women & 12.1 & 11.7 to 12.5 & 1.26 & 1.20 to 1.33 & 10.7 & 10.4 to 11.1 & 1.23 & 1.17 to 1.31 & 1.4 & 1.3 to 1.5 & 1.51 & 1.27 to 1.79 \\
\hline Age & & & & & & & & & & & & \\
\hline 20 to 39 & 1.5 & 1.3 to 1.7 & 1.00 & Reference & 1.4 & 1.2 to 1.6 & 1.00 & Reference & 0.07 & 0.04 to 0.14 & 1.00 & Reference \\
\hline 40 to 49 & 4.7 & 4.3 to 5.1 & 3.17 & 2.65 to 3.78 & 4.4 & 4.0 to 4.8 & 3.12 & 2.60 to 3.74 & 0.3 & 0.2 to 0.4 & 4.11 & 1.88 to 8.99 \\
\hline 50 to 59 & 11.0 & 10.5 to 11.6 & 7.47 & 6.35 to 8.78 & 10.1 & 9.6 to 10.7 & 7.21 & 6.11 to 8.51 & 0.9 & 0.7 to 1.1 & 12.47 & 6.07 to 25.62 \\
\hline 60 to 69 & 18.4 & 17.6 to 19.2 & 12.43 & 10.61 to 14.56 & 16.4 & 15.6 to 17.2 & 11.67 & 9.92 to 13.74 & 2.0 & 1.7 to 2.3 & 27.05 & 13.33 to 54.92 \\
\hline 70 to 79 & 25.1 & 23.9 to 26.3 & 16.97 & 14.47 to 19.91 & 21.6 & 20.5 to 22.8 & 15.41 & 13.07 to 18.17 & 3.4 & 3.0 to 4.0 & 47.19 & 23.25 to 95.78 \\
\hline$\geq 80$ & 24.8 & 23.0 to 26.7 & 16.82 & 14.19 to 19.93 & 21.0 & 19.3 to 22.8 & 14.96 & 12.53 to 17.86 & 3.8 & 3.1 to 4.8 & 52.87 & 25.59 to 109.23 \\
\hline
\end{tabular}

Table 3 Prevalence of any impaired glucose metabolism (IGM), impaired fasting glucose (IFG), impaired glucose tolerance (IGT), and diabetes, overall and by sex and age group, among 2559 participants with a Finnish Diabetes Risk Score (FINDRISC) $\geq 15$ and no previously known diabetes

\begin{tabular}{|c|c|c|c|c|c|c|c|c|}
\hline & \multicolumn{2}{|l|}{ IGM } & \multicolumn{2}{|l|}{ IFG } & \multicolumn{2}{|l|}{ IGT } & \multicolumn{2}{|l|}{ Diabetes } \\
\hline & $\begin{array}{l}\text { Prevalence, \% } \\
(95 \% \mathrm{Cl})\end{array}$ & $\begin{array}{l}\text { Prevalence ratio } \\
(95 \% \mathrm{Cl})\end{array}$ & $\begin{array}{l}\text { Prevalence, \% } \\
(95 \% \mathrm{Cl})\end{array}$ & $\begin{array}{l}\text { Prevalence ratio } \\
(95 \% \mathrm{Cl})\end{array}$ & $\begin{array}{l}\text { Prevalence, \% } \\
(95 \% \mathrm{Cl})\end{array}$ & $\begin{array}{l}\text { Prevalence ratio } \\
(95 \% \mathrm{Cl})\end{array}$ & $\begin{array}{l}\text { Prevalence, \% } \\
(95 \% \mathrm{Cl})\end{array}$ & $\begin{array}{l}\text { Prevalence ratio } \\
(95 \% \mathrm{Cl})\end{array}$ \\
\hline Overall & 34.9 (33.1 to 36.8 ) & & 8.2 (7.2 to 9.3 ) & & $16.9(15.5$ to 18.4$)$ & & 9.8 (8.7 to 11.1$)$ & \\
\hline \multicolumn{9}{|l|}{ Sex } \\
\hline Men & 40.4 (37.4 to 43.4$)$ & 1.00 (Reference) & 9.7 (8.1 to 11.7$)$ & 1.00 (Reference) & 17.9 (15.6 to 20.3$)$ & 1.00 (Reference) & $12.8(10.9$ to 14.9$)$ & 1.00 (Reference) \\
\hline Women & 31.2 (28.9 to 33.6 ) & 0.77 ( 0.70 to 0.86$)$ & 7.1 (5.9 to 8.5 ) & 0.73 (0.56 to 0.94$)$ & $16.2(14.5$ to 18.2$)$ & 0.91 (0.76 to 1.08$)$ & 7.9 (6.6 to 9.3 ) & $0.62(0.49$ to 0.78$)$ \\
\hline \multicolumn{9}{|l|}{ Age, years } \\
\hline 20 to 39 & 19.1 (12.3 to 28.5$)$ & 1.00 (Reference) & 4.3 (1.6 to 11.0$)$ & 1.00 (Reference) & 11.7 (6.5 to 20.1$)$ & 1.00 (Reference) & 3.2 (1.0 to 9.6$)$ & 1.00 (Reference) \\
\hline 40 to 49 & 23.3 (18.6 to 28.9 ) & $1.22(0.76$ to 1.95$)$ & 6.9 (4.4 to 10.7$)$ & $1.62(0.56$ to 4.67$)$ & 10.7 (7.5 to 15.1$)$ & $0.92(0.48$ to 1.77$)$ & 5.7 (3.5 to 9.3 ) & $1.80(0.53$ to 6.08$)$ \\
\hline 50 to 59 & 30.8 (27.3 to 34.6 ) & 1.61 ( 1.05 to 2.48$)$ & $10.1(8.0$ to 12.7$)$ & 2.37 (0.88 to 6.36$)$ & 11.8 (9.5 to 14.6$)$ & $1.01(0.56$ to 1.84$)$ & $8.9(6.9$ to 11.4$)$ & $2.81(0.90$ to 8.80$)$ \\
\hline 60 to 69 & 36.9 (33.6 to 40.2$)$ & 1.93 (1.26 to 2.95$)$ & 9.6 (7.8 to 11.8 ) & 2.25 (0.84 to 6.02$)$ & $16.2(13.8$ to 18.8$)$ & 1.38 (0.78 to 2.46$)$ & 11.1 (9.1 to 13.4$)$ & 3.50 (1.13 to 10.84$)$ \\
\hline 70 to 79 & 38.7 (34.9 to 42.6 ) & 2.02 (1.32 to 3.10$)$ & 6.1 ( 4.5 to 8.4$)$ & 1.44 (0.53 to 3.96$)$ & 22.6 (19.4 to 26.1$)$ & 1.93 (1.09 to 3.43$)$ & 9.9 (7.8 to 12.6$)$ & $3.13(1.00$ to 9.77$)$ \\
\hline$\geq 80$ & 55.5 ( 47.5 to 63.3$)$ & 2.90 ( 1.87 to 4.50$)$ & 5.2 (2.6 to 10.2$)$ & 1.23 (0.38 to 3.97$)$ & 32.7 (25.7 to 40.6 ) & 2.79 (1.53 to 5.09$)$ & 17.6 (12.3 to 24.6 ) & 5.55 (1.73 to 17.80$)$ \\
\hline
\end{tabular}



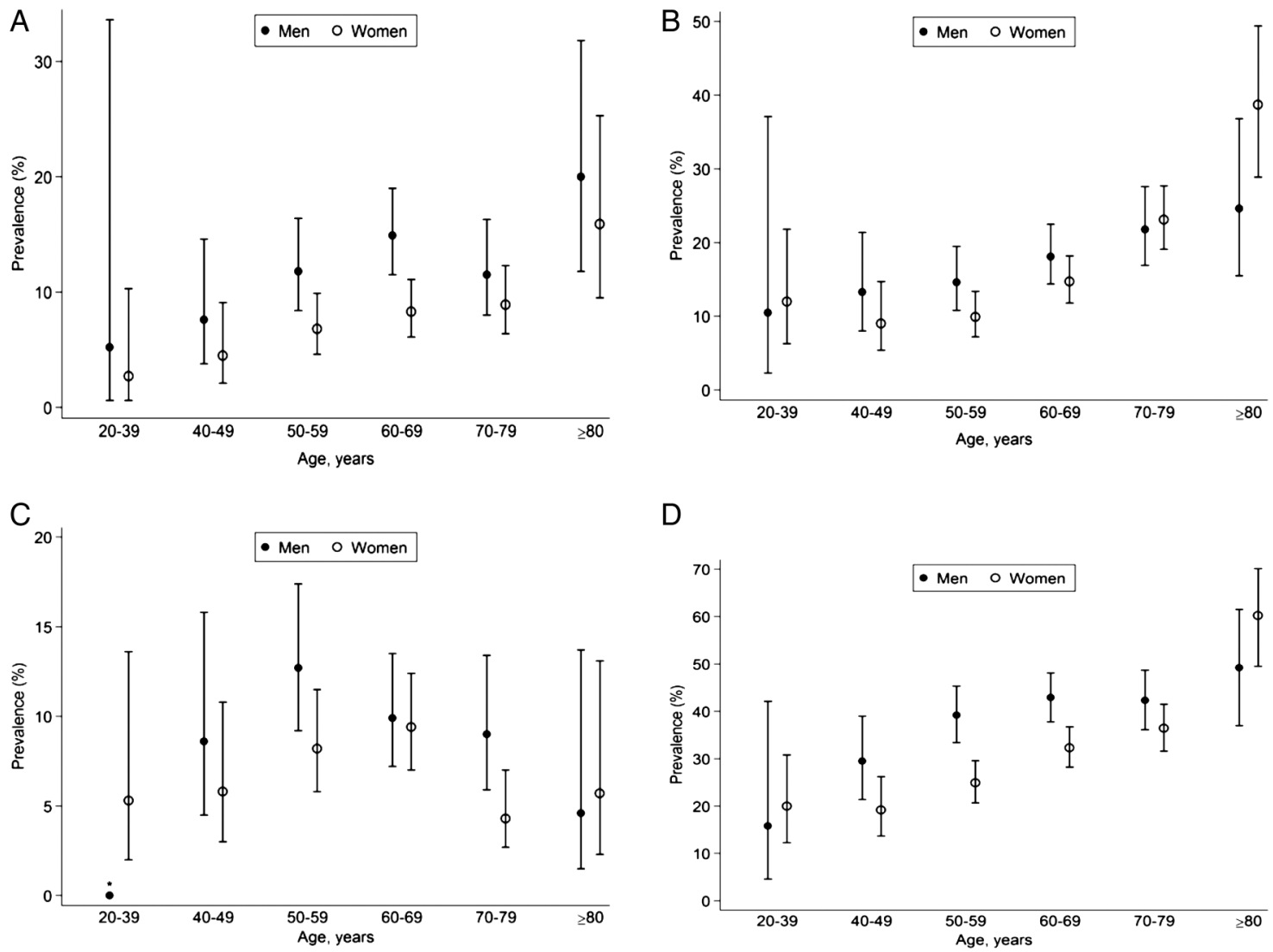

Figure 1 The prevalence of diabetes $(A)$, impaired glucose tolerance $(B)$, impaired fasting glucose (C), and impaired glucose metabolism (D) among those with Finnish Diabetes Risk Score (FINDRISC) $\geq 15$ by different sex and age groups and no previously known diabetes.

\section{CONCLUSIONS}

In this large population-based survey of adults without previously known diabetes, the overall prevalence of elevated FINDRISC $(\geq 15)$ was $11 \%$, and the prevalence increased with age and was higher among women than men. The PPV of elevated FINDRISC was $10 \%$ for prevalent diabetes and $35 \%$ for diabetes and other form of IGM combined. The PPVs were generally higher in men than in women and in older than younger individuals.

The strengths of our study include the populationbased design and the large sample size, which enabled precise estimates across sex and age groups. A limitation is that OGTT was performed among participants with elevated FINDRISC only, and we could not estimate the sensitivity, specificity, and negative predictive value of FINDRISC. Another limitation is that diabetes was classified on the basis of measurements on one occasion, whereas in clinical practice two positive test results are needed to diagnose diabetes among people without symptoms of hyperglycemia. Selection bias cannot be excluded, as the participation rate in the HUNT3 survey was $54 \%$, and $50 \%$ of participants with elevated FINDRISC took part in the HUNT DE-PLAN Study. Reassuringly, the FINDRISC score was similar among participants and non-participants in the HUNT DE-PLAN Study, but non-participants in HUNT3 had a slightly higher prevalence of chronic diseases (including known diabetes), lower socioeconomic status, and higher overall mortality. ${ }^{25}$ Possibly, non-participants may have had a worse glycemic risk profile, which may have led us to underestimate the PPVs of elevated FINDRISC. The participation rate in HUNT3 was lower in younger age groups, ${ }^{20}$ and accordingly the overall prevalence estimate of elevated FINDRISC was slightly attenuated when we corrected for differences in age distribution between HUNT3 participants and the total adult population of Nord-Trøndelag.

Prevalence estimates of elevated FINDRISC similar to ours have been reported from comparable population surveys in Sweden (age range $35-75$ years) ${ }^{16}$ and Finland (age range 45-74 years), ${ }^{8} 19$ and in the non-Hispanic white subpopulation of a survey in the USA. ${ }^{17}$ The PPVs of elevated FINDRISC for prevalent diabetes and IGT in the Swedish study were similar to ours, while the PPV for diabetes was more than twice as high in the American survey and varied from $\sim 10 \%$ to $30 \%$ in sex-specific estimates in the Finnish studies. The PPV for IFG was more than twice as high in the Swedish and Finnish studies ${ }^{8} \quad 1619$ compared with our study. Differences in age distribution, participation rate, or assessment methods for IGM do not appear to explain these differences between studies. 
Several studies in Western Europe, North America, and Australia have reported that men are at higher risk of diabetes than women, ${ }^{26}{ }^{27}$ and that a higher proportion of diabetes cases are undiagnosed in men than in women. ${ }^{8} 171928$ Both these sex differences may contribute to the higher PPVs of elevated FINDRISC in men than in women in our study. However, two studies have reported that at an equal cut-off point for defining elevated FINDRISC, the PPV for prevalent IGM was higher in men and the negative predictive value was higher in women. ${ }^{8} 17$ These findings suggest that the FINDRISC cut-off point of 15 does not imply the same diabetes risk in men as in women. In the original study population where FINDRISC was developed, male sex was recognized as a significant predictor of diabetes, but inclusion of sex in the FINDRISC tool only marginally altered the coefficients of the other score components, and sex was omitted from the scoring system. ${ }^{6}$ Other risk scores, such as the AUSDRISK (Australian type 2 diabetes risk assessment tool) found male sex to be an independent risk factor and therefore included it in the score. ${ }^{28}$ The higher PPV among men than women in our study suggests that inclusion of sex in the FINDRISC score may improve its performance in our population. Further, the strong increase in PPV by age, both overall and within groups having mildly and markedly elevated FINDRISC, suggests that refinement of the age scores may improve the performance of FINDRISC.

If men are at higher risk of diabetes than women, ${ }^{26} 27$ the lower prevalence of elevated FINDRISC in men than in women that we and others ${ }^{8} 1719$ have observed is unexpected. This observation suggests that FINDRISC score components may be underestimated in men relative to women. The components that led to a higher prevalence of elevated FINDRISC among women were a family history of diabetes and previous measurements of high blood glucose and waist circumference. Possibly, the recall or knowledge of a family history of diabetes may be poorer among men than women, ${ }^{29}$ and women go more often to their doctor ${ }^{30} 31$ and may have their glucose level examined more often than men. Also, gestational diabetes provides a warning signal of future diabetes risk that has no counterpart in men. Waist circumference, but not BMI, has increased more in women than in men over the past decades ${ }^{32} 33$ including in this study population, ${ }^{34}$ but how this will influence sex differences in diabetes risk is still not clear.

In this general adult population in Norway, FINDRISC $\geq 15$ classified $11 \%$ as being at high risk for diabetes, and this proportion was higher among women than men. An elevated FINDRISC had a PPV of $10 \%$ for prevalent diabetes and $35 \%$ for diabetes and other forms of IGM combined. The PPV was higher in men than in women and in older than younger individuals. Our data indicate that the impact of sex and age on diabetes risk is not fully captured by FINDRISC, and that refinements to it might improve diabetes prediction.
Author affiliations

${ }^{1}$ Faculty of Medicine, Department of Public Health and General Practice, HUNT Research Centre, NTNU, Norwegian University of Science and Technology,

Levanger, Norway

${ }^{2}$ Dasman Diabetes Insitute, Dasman, Kuwait

${ }^{3}$ Department for Clinical Neurosciences and Preventive Medicine, Danube University Krems, Krems an der Donau, Austria

${ }^{4}$ Chronic Disease Prevention Unit, National Institute for Health and Welfare, Helsinki, Finland

${ }^{5}$ Department of Public Health, University of Helsinki, Helsinki, Finland ${ }^{6}$ Saudi Diabetes Research Group, King Abdulaziz University, Jeddah, Saudi Arabia

${ }^{7}$ Department of Endocrinology, St Olavs Hospital, Trondheim University Hospital, Trondheim, Norway

${ }^{8}$ Unit for Applied Clinical Research, Institute for Cancer Research and Molecular Medicine, Norwegian University of Science and Technology, Trondheim, Norway

${ }^{9}$ Baker IDI, Heart and Diabetes Institute, Melbourne, Australia

Contributors KM planned and organized the data collection in the HUNT DE-PLAN Study. JT planned the European DE-PLAN Study. AJ and BOA analyzed the data, and interpreted the results and wrote the manuscript together with $\mathrm{KM}, \mathrm{JH}$, and SMC. JS interpreted the results and critically reviewed the manuscript. AJ is the guarantor of this work and, as such, had full access to all the data in the study and takes responsibility for the integrity of the data and the accuracy of the data analysis.

Funding The Nord-Trøndelag Health Study (The HUNT Study) is a collaboration between the HUNT Research Centre (Faculty of Medicine, Norwegian University of Science and Technology, NTNU), the Nord-Trøndelag County Council, the Central Norway Health Authority, and the Norwegian Institute of Public Health. The HUNT DE-PLAN Study received financial support from the Norwegian Directorate of Health and the Commission of the European Communities, Directorate C-Public Health, grant agreement no. 2004310.

Competing interests None declared.

Ethics approval Regional Committees for Medical and Health Research Ethics (REK), Norway

Provenance and peer review Not commissioned; externally peer reviewed.

Data sharing statement No additional data are available.

Open Access This is an Open Access article distributed in accordance with the Creative Commons Attribution Non Commercial (CC BY-NC 4.0) license, which permits others to distribute, remix, adapt, build upon this work noncommercially, and license their derivative works on different terms, provided the original work is properly cited and the use is non-commercial. See: http:// creativecommons.org/licenses/by-nc/4.0/

\section{REFERENCES}

1. Guariguata L, Whiting DR, Hambleton I, et al. Global estimates of diabetes prevalence for 2013 and projections for 2035. Diabetes Res Clin Pract 2014;103:137-49.

2. Menke A, Casagrande S, Geiss L, et al. Prevalence of and trends in diabetes among adults in the United States, 1988-2012. JAMA 2015;314:1021-9.

3. Tuomilehto J, Lindstrom J, Eriksson JG, et al. Prevention of type 2 diabetes mellitus by changes in lifestyle among subjects with impaired glucose tolerance. N Engl J Med 2001;344:1343-50.

4. Knowler WC, Barrett-Connor E, Fowler SE, et al. Reduction in the incidence of type 2 diabetes with lifestyle intervention or metformin N Engl J Med 2002;346:393-403.

5. Pan XR, Li GW, Hu YH, et al. Effects of diet and exercise in preventing NIDDM in people with impaired glucose tolerance. The Da Qing IGT and Diabetes Study. Diabetes Care 1997;20:537-44.

6. Lindström J, Tuomilehto J. The diabetes risk score: a practical tool to predict type 2 diabetes risk. Diabetes Care 2003;26:725-31.

7. Paulweber B, Valensi P, Lindstrom J, et al. A European evidence-based guideline for the prevention of type 2 diabetes. Horm Metab Res 2010;42(Suppl 1):S3-36. 
8. Saaristo T, Peltonen M, Lindström J, et al. Cross-sectional evaluation of the Finnish Diabetes Risk Score: a tool to identify undetected type 2 diabetes, abnormal glucose tolerance and metabolic syndrome. Diab Vasc Dis Res 2005;2:67-72.

9. Makrilakis K, Grammatikou S, Liatis S, et al. The effect of a non-intensive community-based lifestyle intervention on the prevalence of metabolic syndrome. The DEPLAN study in Greece. Hormones (Athens, Greece) 2012;11:316-24.

10. Franciosi M, De Berardis G, Rossi MC, et al. Use of the diabetes risk score for opportunistic screening of undiagnosed diabetes and impaired glucose tolerance: the IGLOO (Impaired Glucose Tolerance and Long-Term Outcomes Observational) study. Diabetes Care 2005;28:1187-94.

11. Winkler G, Hidvegi T, Vandorfi G, et al. Prevalence of undiagnosed abnormal glucose tolerance in adult patients cared for by general practitioners in Hungary. Results of a risk-stratified screening based on FINDRISC questionnaire. Med Sci Monit 2013;19:67-72.

12. Garcia-Alcala $\mathrm{H}$, Genestier-Tamborero $\mathrm{CN}$, Hirales-Tamez O, et al. Frequency of diabetes, impaired fasting glucose, and glucose intolerance in high-risk groups identified by a FINDRISC survey in Puebla City, Mexico. Diabetes Metab Syndr Obes 2012;5:403-6.

13. Barengo NC, Acosta T, Arrieta A, et al. Screening for people with glucose metabolism disorders within the framework of the DEMOJUAN project (DEMOnstration area for primary prevention of type 2 diabetes, JUAN Mina and Barranquilla, Colombia). Diabetes Metab Res Rev 2013. Epub ahead of print Sept. 2nd 2013. doi: $10.1002 / \mathrm{dmrr} .2462$

14. Ku GM, Kegels G. The performance of the Finnish Diabetes Risk Score, a modified Finnish Diabetes Risk Score and a simplified Finnish Diabetes Risk Score in community-based cross-sectional screening of undiagnosed type 2 diabetes in the Philippines. Prim Care Diabetes 2013;7:249-59.

15. Soriguer F, Valdes S, Tapia MJ, et al. [Validation of the FINDRISC (FINnish Diabetes RIsk SCore) for prediction of the risk of type 2 diabetes in a population of southern Spain. Pizarra Study]. Med Clin (Barc) 2012;138:371-6.

16. Hellgren Ml, Petzold M, Bjorkelund C, et al. Feasibility of the FINDRISC questionnaire to identify individuals with impaired glucose tolerance in Swedish primary care. A cross-sectional population-based study. Diabet Med 2012;29:1501-5.

17. Zhang L, Zhang Z, Zhang Y, et al. Evaluation of Finnish Diabetes Risk Score in screening undiagnosed diabetes and prediabetes among U.S. adults by gender and race: NHANES 1999-2010. PLoS ONE 2014;9:e97865.

18. Tankova T, Chakarova N, Atanassova I, et al. Evaluation of the Finnish Diabetes Risk Score as a screening tool for impaired fasting glucose, impaired glucose tolerance and undetected diabetes. Diabetes Res Clin Pract 2011:92:46-52.

19. Saaristo T, Moilanen L, Jokelainen J, et al. Cardiometabolic profile of people screened for high risk of type 2 diabetes in a national diabetes prevention programme (FIN-D2D). Prim Care Diabetes 2010;4:231-9.
20. Krokstad S, Langhammer A, Hveem K, et al. Cohort profile: the HUNT study, Norway. Int J Epidemiol 2013;42: 968-77.

21. Schwarz PE, Lindstrom J, Kissimova-Scarbeck K, et al. The European perspective of type 2 diabetes prevention: diabetes in Europe-prevention using lifestyle, physical activity and nutritional intervention (DE-PLAN) project. Exp Clin Endocrinol Diabetes 2008;116:167-72.

22. Alberti KG, Zimmet PZ. Definition, diagnosis and classification of diabetes mellitus and its complications. Part 1: diagnosis and classification of diabetes mellitus provisional report of a WHO consultation. Diabet Med 1998;15:539-53.

23. World Health Organization. Use of Glycated Haemoglobin (HbA1c) in the diagnosis of diabetes mellitus Abbreviated Report of a WHO Consultation, 2011.

24. Statistics Norway. 2015. http://www.ssb.no

25. Langhammer A, Krokstad S, Romundstad P, et al. The HUNT study: participation is associated with survival and depends on socioeconomic status, diseases and symptoms. BMC Med Res Methodol 2012;12:143.

26. Danaei G, Finucane MM, Lu Y, et al. National, regional, and global trends in fasting plasma glucose and diabetes prevalence since 1980: systematic analysis of health examination surveys and epidemiological studies with 370 country-years and 2.7 million participants. Lancet 2011;378:31-40.

27. Wandell PE, Carlsson AC. Gender differences and time trends in incidence and prevalence of type 2 diabetes in Sweden-a model explaining the diabetes epidemic worldwide today? Diabetes Res Clin Pract 2014;106:e90-2.

28. Chen L, Magliano DJ, Balkau B, et al. AUSDRISK: an Australian Type 2 Diabetes Risk Assessment Tool based on demographic, lifestyle and simple anthropometric measures. Med J Aust 2010;192:197-202.

29. Fuentes A, Desrocher M. The effects of gender on the retrieval of episodic and semantic components of autobiographical memory. Memory 2013;21:619-32.

30. GPs and emergency primary health care, 2014. Oslo: Statistics Norway, 2015 [Cited June 14, 2016]. https://www.ssb.no/en/helse/ statistikker/fastlegetj/aar/2015-06-12

31. Nossen JP. Hva foregår på legekontorene? Konsultasjonsstatistikk for 2006. (What happens at the GP' surgeries? Consultation statistics for 2006.) Report No.:4/2007. Oslo: The Norwegian Labour and Welfare Administration; 2007. Norwegian.

32. Ford ES, Maynard LM, Li C. Trends in mean waist circumference and abdominal obesity among US adults, 1999-2012. JAMA 2014;312:1151-3.

33. Janssen I, Shields M, Craig CL, et al. Prevalence and secular changes in abdominal obesity in Canadian adolescents and adults, 1981 to 2007-2009. Obes Rev 2011;12:397-405.

34. Midthjell K, Lee CM, Langhammer A, et al. Trends in overweight and obesity over 22 years in a large adult population: the HUNT Study, Norway. Clin Obes 2013;3:12-20. 